\title{
Expanding the Clinical Phenotype of FGFR1 Internal Tandem Duplication
}

Esko A. Kautto ${ }^{1 ¥}$, Kathleen M. Schieffer ${ }^{1,2 ¥ *}$, Sean McGrath ${ }^{1}$, Anthony R. Miller ${ }^{1}$, Maria Elena HernandezGonzalez $^{1}$, Samantha Choi ${ }^{1}$, Miriam R. Conces ${ }^{2,3}$, Esteban Fernandez-Faith ${ }^{4,5}$, Mai-Lan Ho ${ }^{6,7}$, Kristy Lee ${ }^{1,2,4}$, Anna P. Lillis ${ }^{6,7}$, Gregory D. Pearson ${ }^{8}$, Stephen G. Kaler ${ }^{4,9}$, Richard K. Wilson ${ }^{1,4}$, Elaine R. Mardis ${ }^{1,4,10}$, Vincent Magrini $^{1,3}$, Jeffrey Leonard ${ }^{10,11}$, Catherine E. Cottrell ${ }^{1,2,4}$

${ }^{1}$ The Steve and Cindy Rasmussen Institute for Genomic Medicine, Nationwide Children's Hospital, Columbus, $\mathrm{OH}$, USA

${ }^{2}$ Department of Pathology, The Ohio State University, Columbus, OH, USA

${ }^{3}$ Department of Pathology and Laboratory Medicine, Nationwide Children's Hospital, Columbus, OH, USA

${ }^{4}$ Department of Pediatrics, The Ohio State University, Columbus, OH, USA

${ }^{5}$ Division of Dermatology, Nationwide Children's Hospital, Columbus, OH, USA

${ }^{6}$ Department of Radiology, Nationwide Children’s Hospital Columbus, OH, USA

${ }^{7}$ Department of Radiology, The Ohio State University, Columbus, OH, USA

${ }^{8}$ Department of Pediatric Plastic and Reconstructive Surgery, Nationwide Children’s Hospital, Columbus, OH, USA

${ }^{9}$ Section of Genetic and Genomic Medicine, Nationwide Children's Hospital, Columbus, OH, USA

${ }^{10}$ Department of Neurosurgery, The Ohio State University College of Medicine, Columbus, OH, USA

${ }^{11}$ Division of Neurosurgery, Nationwide Children's Hospital, Columbus, OH, USA

$¥$ These authors contributed equally to this article

\section{*Corresponding Author}

Kathleen M. Schieffer

The Steve and Cindy Rasmussen Institute for Genomic Medicine

Nationwide Children's Hospital

575 Children's Crossroad

Columbus, OH 43215

Phone: 614-355-2894

Email: Kathleen.Schieffer@nationwidechildrens.org

Running title: FGFR1 ITD and spinal dysraphism 


\begin{abstract}
Closed spinal dysraphism (SD) is a type of neural tube defect originating during early embryonic development whereby the neural tissue of the spinal defect remains covered by skin, often coinciding with markers of cutaneous stigmata. It is hypothesized that these events are caused by multifactorial processes, including genetic and environmental causes. We present an infant with a unique congenital midline lesion associated with a closed SD. Through comprehensive molecular profiling of the intraspinal lesion and contiguous skin lesion, an internal tandem duplication (ITD) of the kinase domain of the fibroblast growth factor receptor 1 (FGFR1) gene was found. This ITD variant is somatic mosaic in nature as supported by a diminished variant allele frequency in the lesional tissue, and its absence from the peripheral blood. FGFR1 ITD results in constitutive activation of the receptor tyrosine kinase to promote cell growth, differentiation, and survival through RAS/MAPK signaling. Identification of FGFR1 ITD outside of central nervous system tumors is exceedingly rare and this report broadens the phenotypic spectrum of somatic mosaic FGFR1-related disease.
\end{abstract}




\section{Introduction}

Spinal dysraphism (SD) is a type of neural tube defect that develops during the early embryologic period. The incidence of SD is 0.5-8 cases per 1,000 live births in the United States (Holmes and Li 2019). Two types of neural tube defects can occur: open and closed. Open neural tube defects occur when the brain and/or spinal cord is open to the external environment. In closed SD, a spinal defect is present, but the neural tissue remains covered by skin. Closed neural tube defects occur during early embryologic development due to disruption of neural tube development at various stages. Cutaneous stigmata, including but not limited to hemangioma, lipoma, hyper/hypopigmentation, and/or hypertrichosis are commonly associated and carry a 36\% probability of SD (Holmes and Li 2019; Svokos et al. 2021; Choi et al. 2020). Presence of high-risk cutaneous stigmata warrants additional imaging studies to evaluate for spinal cord injury and the need for neurosurgical intervention (Holmes and Li 2019). SD is thought to occur as the result of multifactorial processes, including both genetic and environmental factors (e.g., nutritional deficiency and maternal obesity). Although few genetic mechanisms have been associated with SD, the biological cause of this developmental event, and in particular the associated cutaneous stigmata, are poorly understood (Salih et al. 2014; Tabatabaei et al. 2020; Greene et al. 2009).

Here, we present an infant with a challenging to diagnose congenital midline hamartomatous lesion. This lesion was found to be associated with a closed SD, arising from the intraspinal compartment and contiguous with the skin. A multidisciplinary clinical workup, in addition to comprehensive molecular profiling, revealed a likely activating somatic mosaic structural rearrangement within the FGFR1 gene.

\section{Results}




\section{Clinical Presentation and Family History}

A female infant was born at an outside hospital by cesarean section due to breech positioning to a healthy mother and father with unremarkable family history. At birth, a $1 \mathrm{~cm}$ diameter midline thoracolumbar skin mass was noted. The mass was firm, and flesh colored with slight white discoloration at the most projecting portion (Fig. 1A), with no signs of tissue breakdown, inflammation or infection noted. The patient's activity and growth parameters were within normal limits. She was found to have rhythmic leg extensions determined by long-term monitoring electroencephalogram to be non-epileptic in nature. A neurosurgical follow-up at our institution at 3 weeks of age noted abnormal lower extremity reflexes and muscle tone. The constellation of findings was concerning for cutaneous stigmata associated with occult spinal dysraphism (SD).

At 10 weeks of age, magnetic resonance imaging (MRI) of the spine showed a large intradural mass spanning the C5-T12 levels, with smooth expansion and remodeling of the bony spinal canal and involvement of the atrophic spinal cord (Fig. 1B). The lesion appeared heterogeneous with interspersed neural, fibrous, vascular, fatty, and hemorrhagic components. Imaging was most suggestive of segmental overgrowth with spinal hamartoma and dorsal dysraphism with overlying cutaneous abnormality. The findings were not characteristic of a true neoplasm given the eccentric medullary origin, native cord atrophy, and associated malformations. A brain MRI was normal for the patient’s age (e.g. normal myelination and sulcation, normal cerebrospinal fluid spaces, no enhancement). A computerized tomography (CT) scan of the head at this time also revealed a narrow and posteriorly ridged sagittal suture, likely suggestive of a developing sagittal synostosis. The anterior fontanelle was open.

Given the MRI findings, the family was referred to dermatology for evaluation of the cutaneous lesion. On physical exam, the lesion was not consistent with a hemangioma or other vascular anomaly. Based on the exam and radiologic findings, it was unclear if the cutaneous lesion was an extension of an underlying spinal disorder 
or a separate cutaneous marker. At 11 weeks of age, the patient underwent resection of the cutaneous lesion and biopsy of the intraspinal mass. Histopathology identified the cutaneous lesion as a stromal and vascular lesion with intermixed nerves and smooth muscle, and the intraspinal mass as a stromal lesion with intermixed fat and fibrous tissue (Fig. 1C and 1D). Immunohistochemistry performed on the cutaneous lesion showed CD31 and ERG positivity in the superficial vascular proliferation and scattered vessels and no immunoreactivity for GLUT-1. A subset of the spindle/stellate cells within fibrous regions were positive for D2-40 and negative for SMA and CD34; however, clusters of glomus cells were positive for SMA and calponin. Desmin and GFAP were negative. S-100 highlighted scattered nerves, and beta-catenin demonstrated a cytoplasmic staining pattern. Additionally, INI-1 and SMARCA4/BRG immunostains were performed and demonstrated retained weak to moderate nuclear staining. The histologic features were not diagnostic of a distinct entity. Hemangioma or other vascular anomaly was considered, but this diagnosis was felt less likely given the prominent stromal component and overall characteristics of the lesion. The triphasic morphology of fibrous hamartoma of infancy was not present. There were no histologic features present to support a diagnosis of teratoma or low-grade glioma.

Immediately following the surgical biopsies, the patient demonstrated limited, predominately reflexive, movement in the lower extremities. She was referred for physical and occupational therapy. Three months postbiopsy (5 months of age), she demonstrated occasional spontaneous movements in the lower extremities which remained decreased relative to the upper extremities. There was absence of sensory response at the thoracic region. At 6.5 months of age, the patient was seen by clinical genetics for counseling to better inform the parents of recurrence risk for future offspring. Her head shape was slightly dolichocephalic, and the anterior fontanelle was prematurely closed. Congenital dermal melanocytosis and mild sacral hirsutism were noted on the back, in addition to a well-healed midline thoracolumbar scar with several prominent skin folds on the left back. The neurological examination showed normal truncal strength and tone, and no head lag on pull-to-sit. 
She smiled, cooed, and fixed and tracked visually. Her arms were active and used to push up and to lift her head from the prone position. The legs were flaccid with no stepping reflex or weight-bearing noted. Deep tendon reflexes were present in all limbs. Intermittent clonus (3-4 beats) was noted at the left ankle.

She was followed in our myelomeningocele clinic and is undergoing physical therapy with the goal of developing age-appropriate upper body motor skills. MRI at 12 months of age described an unchanged appearance of the residual intraspinal mass suggestive of a hamartomatous malformation, as could be seen in the spectrum of PIK3CA-related overgrowth syndrome (PROS). However, segmental overgrowth or macrocephaly was not appreciated. MRI at 15 months demonstrated progressive change of the lesion with interval expansion, particularly of the caudal portion. Developmentally, the patient can roll, prop sit independently, and belly crawl. She is vocalizing and uses simple words. At 17 months, the patient was seen at an outside hospital for consultation, where a spinal MRI revealed an increase in size of the intraspinal lesion with mass effect on the spinal cord. Given these findings, sirolimus, an mTOR inhibitor, was initiated.

\section{Genomic Analyses}

Given the absence of a definitive diagnosis, the patient was enrolled on an Institutional Review Board approved translational research protocol which performs comprehensive molecular profiling, including paired somatic disease/germline comparator exome sequencing and RNA sequencing of disease-involved samples. Molecular profiling of the disease-involved intraspinal lesion and the contiguous cutaneous lesion was performed, while peripheral blood was used as the comparator germline sample (Supplemental Methods, Supplemental Table 1). Enhanced exome sequencing (eES) data analysis did not initially reveal any clearly medically meaningful germline or somatic single nucleotide variants, small insertion/deletion events or copy number variants (CNVs) associated with disease. 
However, analysis of short-read RNA-sequencing data revealed an internal tandem duplication (ITD) of FGFR1 (NM_023110.3) exons 10-18 ( 5.2kb), with intervening sequence derived from intron 9 joining the duplicated segments and maintaining translational reading frame (Fig. 2, Supplemental Fig. 1A). An alternative sequencing approach enabling the detection of isoforms using Pacific Biosciences Iso-Seq methodology allowed for orthogonal verification of an expressed FGFR1 gene product harboring the ITD (Supplemental Methods). Retrospective manual review of the eES data provided visual evidence of the FGFR1 alteration in the cutaneous lesion (20 out of 312 reads, 6.4\%) and the intraspinal mass (27 out of 288 reads, 9.4\%), with no evidence detected in the comparator blood sample (Supplemental Fig. 1B). Furthermore, Pacific Biosciences HiFi wholegenome, long-read sequencing was performed, demonstrating the presence of the FGFR1 ITD in 8 out of 33 reads (24.2\%). The reduced variant allele frequency is consistent with a non-heterozygous state, supporting somatic mosaic etiology. Differences in the reported variant allele frequencies between short-read exome and HiFi long-read genome sequencing can be expected. With short-read sequencing, an inherent challenge exists in split alignments of short sequence spans across a structural variant boundary. Thus, the influence of read alignment and soft clipping in the setting of structural variation may lead to an under-representation of ITDsupporting reads and concomitantly, a lower variant allele frequency. Whereas long-read sequencing, such as that used in Pacific Biosciences HiFi, may enable representation of the complete structural variant in a single read preserving order and orientation of the genomic alteration. Precise boundaries for the duplication were obtained from the HiFi data, with 5,259 nucleotides of FGFR1 (NM_023110.3) duplicated, starting in intron 9 and ending at amino acid 819 in exon 18. This ITD results in a duplication of the tyrosine kinase domain (amino acids 464-765) and is predicted to be an activating event (Fig. 2). The FGFR1 ITD was clinically confirmed by reverse transcriptase-PCR followed by Sanger sequencing in our CLIA-certified laboratory (Supplemental Fig. 1C). 


\section{Discussion}

Herein, we present an infant with an unusual cutaneous thoracic lesion, initially considered by physical exam to possibly represent an isolated congenital hemangioma. However, cross-sectional imaging revealed a segmental intradural mass and SD with resulting spinal cord involvement and compression. Although multidisciplinary clinical evaluation and histopathology was non-diagnostic, comprehensive molecular profiling of diseaseinvolved tissue revealed a mosaic activating FGFR1 ITD suggestive of a novel biological mechanism associated with SD development.

Closed SD may be associated with spinal cord tethering or compression which may result in significant neurologic, orthopedic, and urologic morbidity. Clinical features, including the presence of a congenital midthoracic lesion or high-risk cutaneous stigmata, should warrant additional imaging studies and neurosurgical evaluation is crucial to aid in supportive management (Holmes and Li 2019; Svokos et al. 2021; Choi et al. 2020). In our described case, the non-epileptic rhythmic leg extensions seen in early perinatal life along with abnormal lower extremity reflexes and muscle tone were suggestive of morbidity due to this lesion. Development of neural tube defects is multifactorial, likely involving both genetic and environmental factors (Netto et al. 2009). In this family, there was no evidence of known environmental factors associated with neural tube defects during pregnancy. Our patient also presented with slight dolichocephaly, premature closing of the anterior fontanelle, and a developing sagittal synostosis. The FGFR gene family is associated with germline craniosynostosis disorders, and familial sagittal synostosis with scaphocephaly has been reported in association with an FGFR2 missense variant in a single family (McGillivray et al. 2005). Dolichocephaly has also been described in infants born with breech presentation, which may contribute to the phenotypic features seen in our patient (Kasby and Poll 1982; Haberkern et al. 1979). 
Genetic factors may contribute to the etiology of SD. However, the biological mechanisms underlying this developmental anomaly and associated cutaneous stigmata are not well understood and may be under-reported due to inherent challenges in resolving these genetic events. Post-zygotic variation, including single nucleotide changes and copy number alteration, has been described in planar cell polarity genes in association with open neural tube defects (Tian et al. 2020; Galea et al. 2021). Such findings support that somatic mosaicism may serve as a mechanism of disease. However, study of disease involved tissue may be critical, in addition to use of sensitive methods to resolve low frequency and/or structurally complex variation. Recently, exome sequencing of genomic DNA from two individuals with SD and hamartomatous lesions was not revealing for an underlying genomic event (Bartels et al. 2020). Limiting sample size ( $n=2)$, and the tissue under study may have been contributory to the inability to establish a genetic association with disease. In addition to the criticality in studying disease-involved tissues, the use of short-read sequencing and the associated software algorithms to resolve genomic variation may be limiting due to assay sensitivity or the challenge in accurately aligning reads. Therefore, it is reasonable to hypothesize that mosaic variation, and in particular, complex structural mosaic variation, may currently be under-reported in the literature due to technical limitations of the assays most commonly used for clinical diagnostics and research. In our described patient, the intraspinal mass and contiguous skin lesion were found to harbor an ITD in the FGFR1 gene. FGFR1 encodes fibroblast growth factor receptor 1, a receptor tyrosine kinase (RTK) that mediates signaling through the RAS/MAPK signaling pathways to regulate cell growth, differentiation, survival, and angiogenesis. This FGFR1 ITD results in duplication of $\sim 5 \mathrm{~kb}$ of genomic sequence oriented one segment in front of the other. Identifying such events is bioinformatically challenging using short read sequencing methodology with read lengths typically in the range of 50-250bp. Due to this challenge of resolving larger structural variants (SV), long-read sequencing based translational assays were utilized to further define the molecular characteristics of the lesion. Long-read sequencing methodologies have greatly improved in accuracy, enabling mapping of kilobase sequence reads and facilitating the study of full-length mRNA transcripts and genomic segments. These long reads make it 
possible to precisely resolve SV breakpoints found challenging when using Illumina-based short-read sequencing (Wenger et al. 2019).

Pediatric malignancies, including teratoma, soft tissue sarcomas, and low-grade gliomas were initially considered but ruled out by integrative evaluation, including radiology and pathology. Somatic alterations in FGFR1 have been observed across multiple pediatric CNS tumors, including pilocytic astrocytomas, low-grade gliomas, and dysembryoplastic neuroepithelial tumors (Ryall et al. 2020; Zhang et al. 2013; Fina et al. 2017; Jones et al. 2013; Ardizzone et al. 2020). FGFR1 ITD has been described in low-grade gliomas, among a spectrum of other FGFR1 genomic alterations, including single nucleotide variants, gene fusions, and copy number alterations (Ryall et al. 2020; Zhang et al. 2013; Fina et al. 2017; Jones et al. 2013; Uchiyama et al. 2021). Notably, the described events are consistent with a gain-of-function mechanism. FGFR1 activating missense variants have been described in encephalocraniocutaneous lipomatosis (ECCL), a sporadic condition characterized by anomalies in the cutaneous, ocular, and central nervous systems (Moog 2009; Bennett et al. 2016). In ECCL, the FGFR1 variants are somatic mosaic in nature, affecting only the involved tissues and arising from post-zygotic mitotic error. While the somatic mosaic etiology of the genomic alteration is consistent with that seen in our patient, she does not fulfill the diagnostic clinical criteria for ECCL (Moog 2009). Our described patient did not present with the hallmark feature of ECCL, naevus psiloliparus, a fatty tissue naevus of the scalp with variable degree of alopecia, nor did she present with facial tags or lipomas, facial asymmetry, alopecia, skeletal/jaw lesions, or ventriculomegaly (Moog 2009). Although she has not been seen by ophthalmology, a review of systems exam identified no ocular or vision abnormalities. CNS lipomas are also a common feature among individuals with ECCL (33/54 cases), with intracranial lipomas being more common than intraspinal (Moog 2009). These lipomas typically present as collections of bulk fat without associated soft tissue or vascularity, involving the head and neck in a unilateral or asymmetric distribution with intracranial or skin lipomas, ocular dermolipomas, possible brain overgrowth/tumors, and occasionally bone cysts/tumors. The 
intraspinal mass seen in our patient is phenotypically distinct from the intraspinal lipomas described in individuals with ECCL, demonstrating hamartomatous overgrowth, including disorganized bulk fat, soft tissue, fibrous tissue, and vessels. Given the unique manifestation, this may serve to broaden the phenotypic spectrum of somatic mosaic FGFR1-related disorders.

As discerned through study of CNS tumors, FGFR1 ITD results in auto-phosphorylation of FGFR1 and downstream p-ERK1/2, consistent with constitutive pathway activation (Zhang et al. 2013; Jones et al. 2013). The FGF pathway is also critical during embryologic development, with in vivo disruption of FGFR1 in particular, demonstrating aberrant neurulation and a spina bifida phenotype in mice (Deng et al. 1997; Hoch and Soriano 2006). Within our described patient, given the absence of the FGFR1 ITD in the peripheral blood and the reduced variant frequency in affected tissues, a post-zygotic mitotic event resulting in somatic mosaicism is the proposed etiology. Thus, one may hypothesize that if the post-zygotic error resulting in FGFR1 ITD occurred early in embryologic development, the disruption of tightly regulated signaling may serve as a genetic driver of SD with cutaneous stigmata. Somatic mosaicism of genes involved in the RAS/MAPK signaling pathway have been implicated in numerous overgrowth and vascular disorders with cutaneous features, including PROS and CLOVES (Greene and Goss 2018). Interestingly, our patient’s MRI demonstrated features consistent with a hamartomatous lesion, as described in the spectrum of PROS. While the genomic event underlying PROS differs from that seen in our patient, dysregulation of the downstream pathway (RAS/MAPK) is a shared feature. Furthermore, it is notable that small duplications have been previously documented in RTK genes in association with hamartomatous lesions. Such has been described in fibrous hamartoma of infancy, with a characteristic triphasic morphology consisting of fibrocollagenous tissue, primitive mesenchymal cells, and mature adipose tissue, in association with somatic, activating EGFR exon 20 duplication/insertion events (Park et al. 2016). Commonalities in the etiology of the aforementioned conditions, which include activating 
somatic variation and dysregulation of cellular signaling pathways, lend further credence to the association of our patient's clinical features and the described FGFR1 alteration.

FGFR inhibitors are under clinical investigation in the setting of FGFR-altered somatic disease (Ardizzone et al. 2020). Several FGFR inhibitors are now FDA-approved (pemigatinib and infigratinib) in the setting of unresectable advanced or metastatic cholangiocarcinoma with FGFR2 fusion or rearrangement, and erdafitinib for advanced or metastatic bladder cancer with FGFR2 or FGFR3 alteration (fda.gov). Translation of FGFR inhibitors for utility in germline disease is still in preclinical stages, as in vitro and in vivo studies targeting the FGFR craniosynostosis disorders have demonstrated some success in preventing premature fusion of sutures (Rachwalski et al. 2019).

In summary, we present the complex history of a female infant with a congenital midthoracic skin lesion in the setting of a closed SD. Given the unique presentation, molecular analysis of the intradural mass and contiguous skin lesion was performed to better inform disease etiology. Ultimately, an integrative analysis and multidisciplinary approach to this patient's care identified a novel disease association in the setting of an FGFR1 ITD. Detection of the underlying genomic alteration informed monitoring and surveillance for this patient and elucidated the molecular pathogenesis associated with this atypical cutaneous stigmata of closed SD.

\section{Methods}

The patient was enrolled as part of an Institutional Review Board (IRB) approved study (IRB17-00206) at The Steve and Cindy Rasmussen Institute for Genomic Medicine (IGM) at Nationwide Children’s Hospital (NCH). Written informed consent was provided for comprehensive genomic analysis. Peripheral blood was collected by routine venipuncture for genomic DNA extraction. Snap frozen tissue from the skin and intraspinal lesions were obtained for DNA and RNA extraction. Comprehensive molecular analysis, including paired somatic 
disease/comparator germline exome sequencing, whole transcriptome sequencing of the disease-involved tissue, and Pacific Biosciences Single Molecule Real Time (SMRT) sequencing-based methodologies were performed as described in the Supplemental Methods (sequencing metrics are described in Supplemental Table 1).

\section{Data availability statement}

The datasets generated during and/or analyzed during the current study are available in dbGAP (https://www.ncbi.nlm.nih.gov/gap/) [phs001820.v1.p1]. The FGFR1 ITD has been deposited in ClinVar under the accession number SCV002072498.

\section{Patient consent for publication}

Written informed consent was provided by the patient’s parents for publication.

\section{Ethics approval}

The patient was enrolled as part of an Institutional Review Board (IRB) approved study (IRB17-00206) at The Steve and Cindy Rasmussen Institute for Genomic Medicine (IGM) at Nationwide Children’s Hospital (NCH).

\section{Acknowledgements}

We thank the patient and their family for participating in our translational research protocol.

\section{Author contributions}

KMS, RKW, ERM, VM, CEC conceptualized the study and provided methodology; MRC provided histopathological workup; EFF, GDP, SGK and JL provided clinical workup, MLH and APL provided radiologic workup, EAK, KMS, SM, ARM, MEHG, SC, CEC were involved in consent, analysis, and 
interpretation of next generation sequencing data and PacBio data, EAK and KMS drafted the first version of the manuscript; all authors reviewed and agreed on the final version of the manuscript.

\section{Funding}

We thank the Nationwide Foundation Pediatric Innovation Fund for generously supporting sequencing, data production, and analysis. E.A.K. was supported by the National Institute of General Medical Sciences of the National Institutes of Health under Award Number 2T32GM068412-11A1. The content is solely the responsibility of the authors and does not necessarily represent the official views of the National Institutes of Health.

\section{Competing Interests}

The authors declare that there are no competing interests.

\section{References}

Ardizzone A, Scuderi SA, Giuffrida D, Colarossi C, Puglisi C, Campolo M, Cuzzocrea S, Esposito E, Paterniti I. 2020. Role of Fibroblast Growth Factors Receptors (FGFRs) in Brain Tumors, Focus on Astrocytoma and Glioblastoma. Cancers (Basel) 12: 1-22. doi: 10.3390/cancers12123825

Bartels RHMA, Grotenhuis JA, Stegmann APA, Brunner H. 2020. Genetic analysis of spinal dysraphism with a hamartomatous growth (appendix) of the spinal cord: a case series. BMC Neurol 2020 20: 1-4. doi:

10.1186/s12883-020-01710-7 
Bennett J, Tan T, Alcantara D, Tétrault M, Timms A, Jensen D, Collins S, Nowaczyk M, Lindhurst M, Christensen K, et al. 2016. Mosaic Activating Mutations in FGFR1 Cause Encephalocraniocutaneous Lipomatosis. Am J Hum Genet 98: 579-587. doi: 10.1016/j.ajhg.2016.02.006

Choi SJ, Yoon HM, Hwang JS, Suh CH, Jung AY, Cho YA, Lee JS. 2020. Incidence of Occult Spinal Dysraphism Among Infants With Cutaneous Stigmata and Proportion Managed With Neurosurgery: A Systematic Review and Meta-analysis. JAMA Netw Open 3: e207221.

Deng C, Bedford M, Li C, Xu X, Yang X, Dunmore J, Leder P. 1997. Fibroblast growth factor receptor-1 (FGFR-1) is essential for normal neural tube and limb development. Dev Biol 185: 42-54. doi: 10.1006/dbio.1997.8553

Fina F, Barets D, Colin C, Bouvier C, Padovani L, Nanni-Metellus I, Ouafik L, Scavarda D, Korshunov A, Jones DTW, et al. 2017. Droplet digital PCR is a powerful technique to demonstrate frequent FGFR1 duplication in dysembryoplastic neuroepithelial tumors. Oncotarget 8: 2104-2113. doi: 10.18632/oncotarget.12881

Galea G, Maniou E, Edwards T, Marshall A, Ampartzidis I, Greene N, Coppe A. 2021. Cell non-autonomy amplifies disruption of neurulation by mosaic Vangl2 deletion in mice. Nat Commun 12: 1159. doi: 10.1038/s41467-021-21372-4

Greene AK, Goss JA. 2018. Vascular Anomalies: From A Clinicohistologic to a Genetic Framework. Plast Reconstr Surg 141: 709e. doi: 10.1097/PRS.0000000000004294 
Greene NDE, Stanier P, Copp AJ. 2009. Genetics of human neural tube defects. Hum Mol Genet 18: R113. doi: 10.1093/hmg/ddp347

Haberkern CM, Smith DW, Jones KL. 1979. The "breech head” and its relevance. Am J Dis Child 133: 154156. doi: 10.1001/archpedi.1979.02130020044009

Hoch R, Soriano P. 2006. Context-specific requirements for Fgfr1 signaling through Frs2 and Frs3 during mouse development. Development 133: 663-673. doi: 10.1242/dev.02242

Holmes L, Li V. 2019. Occult Spinal Dysraphism. Pediatr Rev 40: 650-652. doi: 10.1542/pir.2018-0155

Jones DTW, Hutter B, Jäger N, Korshunov A, Kool M, Warnatz HJ, Zichner T, Lambert SR, Ryzhova M, Quang DAK, et al. 2013. Recurrent somatic alterations of FGFR1 and NTRK2 in pilocytic astrocytoma. Nat Genet 45: 927-932. doi: 10.1038/ng.2682

Kasby CB, Poll V. 1982. The breech head and its ultrasound significance. Br J Obstet Gynaecol 89: 106-110. doi: 10.1111/j.1471-0528.1982.tb04674.x

McGillivray G, Savarirayan R, Cox TC, Stojkoski C, McNeil R, Bankier A, Bateman JF, Roscioli T, Gardner RJM, Lamandé SR. 2005. Familial scaphocephaly syndrome caused by a novel mutation in the FGFR2 tyrosine kinase domain. J Med Genet 42: 656-662. doi: 10.1136/jmg.2004.027888

Moog U. 2009. Encephalocraniocutaneous lipomatosis. J Med Genet 46: 721-729. doi:

10.1136/jmg.2009.066068 
Netto JMB, Bastos AN, Figueiredo AA, Pérez LM. 2009. Spinal Dysraphism: A Neurosurgical Review for the Urologist. Rev Urol 11: 71.

Park J, Cohen C, Lopez D, Ramos E, Wagenfuehr J, Rakheja D. 2016. EGFR Exon 20 Insertion/Duplication Mutations Characterize Fibrous Hamartoma of Infancy. Am J Surg Pathol 40: 1713-1718. doi:

10.1097/PAS.0000000000000729

Rachwalski M, Khonsari RH, Paternoster G. 2019. Current Approaches in the Development of Molecular and Pharmacological Therapies in Craniosynostosis Utilizing Animal Models. Mol Syndromol 10: 115-123. doi: $10.1159 / 000493535$

Ryall S, Zapotocky M, Fukuoka K, Nobre L, Guerreiro Stucklin A, Bennett J, Siddaway R, Li C, Pajovic S, Arnoldo A, et al. 2020. Integrated Molecular and Clinical Analysis of 1,000 Pediatric Low-Grade Gliomas. Cancer Cell 37: 569-583.e5. doi: 10.1016/j.ccell.2020.03.011

Salih MA, Murshid WR, Seidahmed MZ. 2014. Classification, clinical features, and genetics of neural tube defects. Saudi Med J 35: S5-S14.

Svokos K, Batista-Silverman L, Graber S, O’Neill B, Handler M. 2021. International survey on the management of lumbosacral cutaneous stigmata in infants with suspected occult spinal dysraphism. $J$ Neurosurg Pediatr 28: 592-599. doi: 10.3171/2021.5.PEDS2126 
Tabatabaei RS, Fatahi-Meibodi N, Meibodi B, Javaheri A, Abbasi H, Hadadan A, Bahrami R, Mirjalili SR, Karimi-Zarchi M, Neamatzadeh H. 2020. Association of Fetal MTHFR C677T Polymorphism with Susceptibility to Neural Tube Defects: A Systematic Review and Update Meta-Analysis. Fetal Pediatr Pathol 1-17 doi: 10.1080/1551381520201775734

Tian T, Lei Y, Chen Y, Karki M, Jin L, Finnell R, Wang L, Ren A. 2020. Somatic mutations in planar cell polarity genes in neural tissue from human fetuses with neural tube defects. Hum Genet 139: 1299-1314. doi: 10.1007/s00439-020-02172-0

Uchiyama T, Gomi A, Nobusawa S, Fukushima N, Matsubara D, Kawai K. 2021. A case of a rosette-forming glioneuronal tumor with clinicopathological features of a dysembryoplastic neuroepithelial tumor and fibroblast growth factor receptor 1 internal tandem duplication. Brain Tumor Pathol 38: 250-256. doi:10.1007/s10014021-00397-z

Wenger AM, Peluso P, Rowell WJ, Chang PC, Hall RJ, Concepcion GT, Ebler J, Fungtammasan A, Kolesnikov A, Olson ND, et al. 2019. Accurate circular consensus long-read sequencing improves variant detection and assembly of a human genome. Nat Biotechnol 37: 1155-1162. doi: 10.1038/s41587-019-0217-9

Zhang J, Wu G, Miller CP, Tatevossian RG, Dalton JD, Tang B, Orisme W, Punchihewa C, Parker M, Qaddoumi I, et al. 2013. Whole-genome sequencing identifies genetic alterations in pediatric low-grade gliomas. Nat Genet 45: 602-612. doi: 10.1038/ng.2611 


\section{Figure Legends}

Figure 1: Visualization of Lesion. A) At birth, a $1 \mathrm{~cm}$ diameter firm and flesh colored skin mass with slight white discoloration was identified at the midline thoracolumbar region. B) Radiologic visualization of lesion at 10 weeks of age pre-surgical intervention. Spine MRI with sagittal and axial T1, T2 with fat suppression, and postcontrast T1 with fat suppression. Large intradural mass (brackets) spans the C5-T12 levels, with smooth expansion and remodeling of the bony spinal canal. This partially encases, compresses, and is integrated with the native spinal cord, which appears atrophic. The lesion consists of disorganized neural and fibrous tissue, enhancing vessels, bulk fat, and hemorrhage. There is a midthoracic dorsal spinal dysraphism with overlying cutaneous enhancing lesion (arrows). C) Histopathology of the cutaneous lesion involved the dermis and subcutaneous adipose tissue and was composed of stromal and vascular components. The variably cellular stroma contained cells with round to elongated nuclei, no cytologic atypia, and inconspicuous cytoplasm. D) The vascular component was composed predominantly of scattered capillary channels.

Figure 2: Schematic diagram of the FGFR1 Internal Tandem Duplication (ITD). A) The exon structure of the wildtype FGFR1 NM_023110.3 transcript and the associated NP_075598.2 protein structure. The protein kinase domain (amino acids 464-765), colored in orange, originates from sequence of exons 10-18. B) The exon structure of NM_023110.3 with the observed 5,259 bp ITD, spanning from intron 9 into exon 18. This genomic alteration results in a full, in-frame duplication of the protein kinase domain. 
Table 1:

\begin{tabular}{|c|c|c|c|c|c|c|}
\hline Gene & Chromosome & $\begin{array}{c}\text { HGVS DNA } \\
\text { Reference }\end{array}$ & $\begin{array}{c}\text { HGVS Protein } \\
\text { Reference }\end{array}$ & $\begin{array}{c}\text { Predicted } \\
\text { Effect }\end{array}$ & Genotype & ClinVar ID \\
\hline FGFR1 & $8 \mathrm{p} 11.23$ & $\begin{array}{c}\text { g.38413642- } \\
\text { (NM_023110.3) }\end{array}$ & p.Val429_Leu829dup & $\begin{array}{c}\text { Internal } \\
\text { Tandem } \\
\text { Duplication }\end{array}$ & $\begin{array}{c}\text { Somatic } \\
\text { mosaic }\end{array}$ & SCV002072498 \\
\hline
\end{tabular}




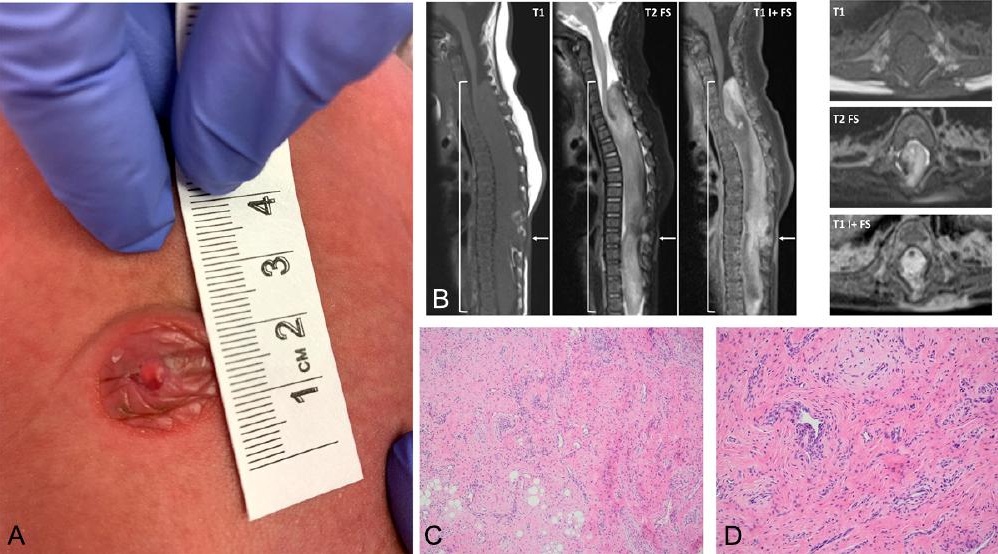


A NM_023110.3 wildtype sequence

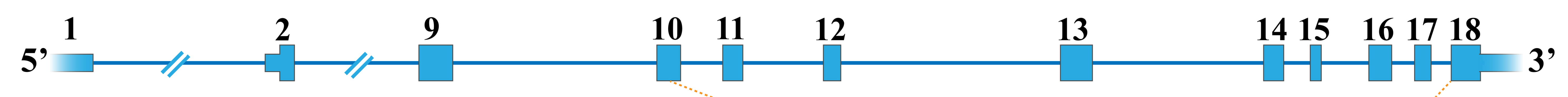

NP_075598.2 protein structure

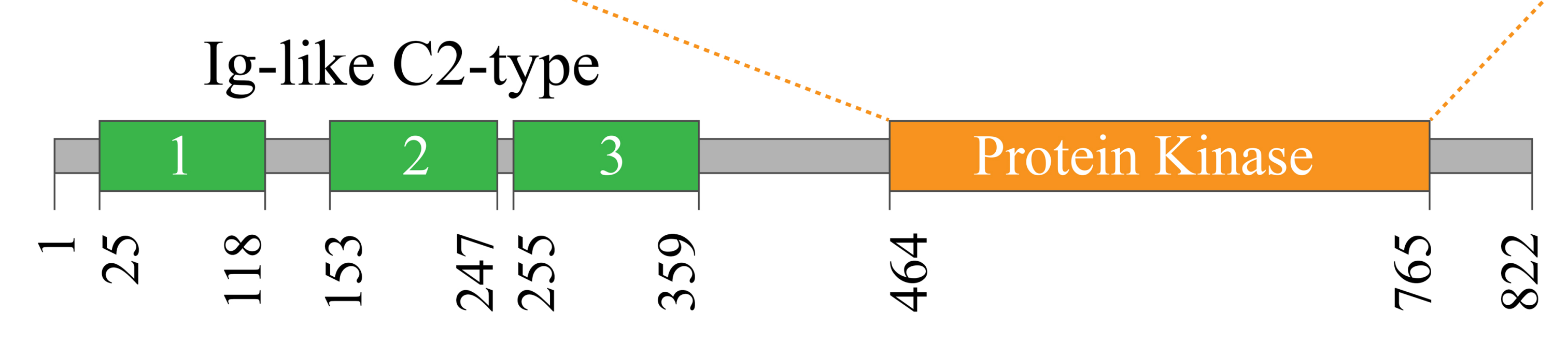

B NM_023110.3 with internal tandem duplication of exons 10-18* (partial exon 18)
5,1

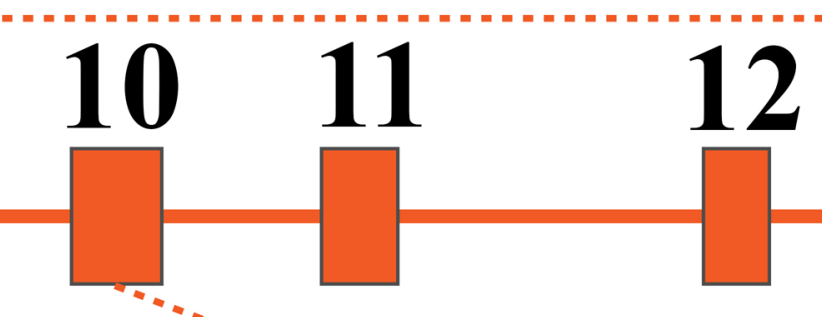
NP_075598.2 w/ duplicated kinase domain

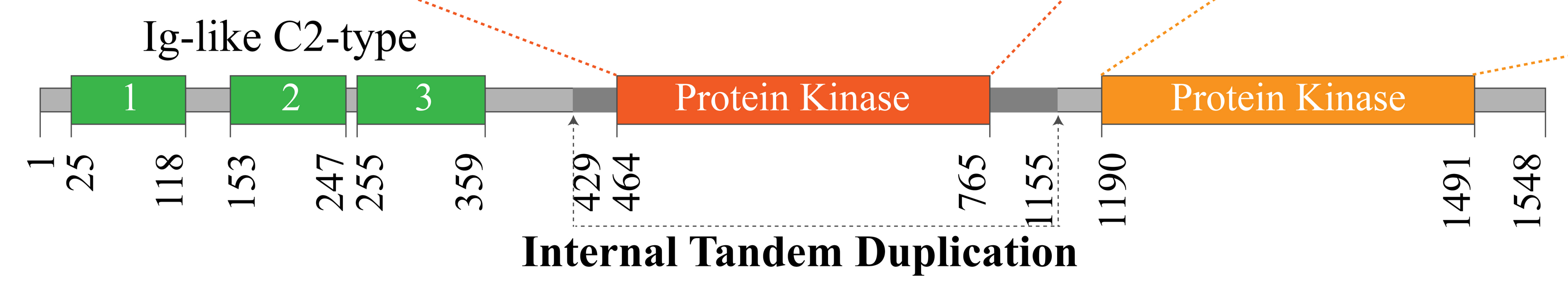

5259 bp duplication 


\section{COLD SPRING HARBOR Molecular Case Studies}

\section{Expanding the Clinical Phenotype of FGFR1 Internal Tandem Duplication}

Esko A Kautto, Kathleen M Schieffer, Sean McGrath, et al.

Cold Spring Harb Mol Case Stud published online February 11, 2022

Access the most recent version at doi: $10.1101 / \mathrm{mcs} . \mathrm{a} 006174$

\section{Supplementary http://molecularcasestudies.cshlp.org/content/suppl/2022/03/09/mcs.a006174.D Material C1}

Published online February 11, 2022 in advance of the full issue.

Accepted Peer-reviewed and accepted for publication but not copyedited or typeset; accepted Manuscript manuscript is likely to differ from the final, published version. Published onlineFebruary 11,2022 in advance of the full issue.

Creative This article is distributed under the terms of the

Commons http://creativecommons.org/licenses/by-nc/4.0/, which permits reuse and License redistribution, except for commercial purposes, provided that the original author and source are credited.

Email Alerting Receive free email alerts when new articles cite this article - sign up in the box at the Service top right corner of the article or click here. 